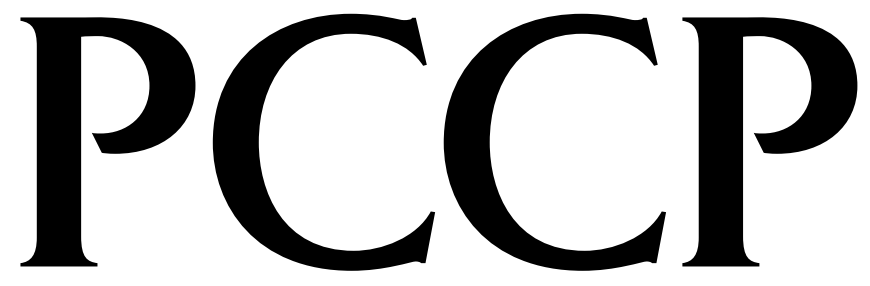

Physical Chemistry Chemical Physics 


\title{
Parametrically polarization shaped pulses guided via a hollow core photonic crystal fiber for coherent control
}

\author{
Fabian Weise,* Georg Achazi and Albrecht Lindinger \\ Received 25th October 2010, Accepted 10th March 2011 \\ DOI: $10.1039 / \mathrm{c0cp02263b}$ \\ We present ultrafast polarization pulse shaping through a micro structured hollow core photonic \\ crystal fiber. The pulses are shaped in pulse sequences in which the energy, distance, phases, and \\ chirps as well as the state of polarization of each individual sub-pulse can be independently \\ controlled. The application of these pulses for coherent control is demonstrated for feedback loop \\ optimization of the multi-photon ionization of potassium dimers. In a second experiment, this \\ process is investigated by shaper-assisted pump-probe spectroscopy which is likewise performed \\ with pulses that are transmitted through the fiber. Both techniques reveal the excitation pathway \\ including the dynamics in the participating electronic states and expose the relevance of the \\ polarization. These methods will be valuable for endoscopic applications.
}

\section{Introduction}

Femtosecond laser pulses have become a popular tool in science and technology. Their application ranges from fundamental research of quantum dynamics to material processing. For many of these applications it could be desirable to transport the pulses with fiber optics. Conventional fibers which guide the light by total internal reflection are unsuitable for pulses due to constraints of the core material. Chromatic dispersion strongly broadens the pulses temporally. This could be overcome by pre-chirping the pulse using prism and grating compressors or adaptive phase control. ${ }^{1}$ Further, the transmission of ultrashort pulses is limited to pulse energies below $0.1 \mathrm{~nJ}$ because non-linear effects such as self-phase modulation become significant. This makes standard optical fibers unsuitable for many applications.

The use of micro structures revolutionized fiber optics. The guiding mechanism in photonic crystal fibers is fundamentally different and relies on a photonic band gap waveguide. ${ }^{2}$ The properties of these fibers are determined by the geometry of the structure and its dimensions. Hollow core photonic crystal fibers are particularly suitable to transport pulses which have a high peak intensity. ${ }^{3}$ The accessible pulse energy is adequate to drive multi-photon processes and extends the area of application. A standard procedure for investigating ultrafast quantum dynamics is pump-probe spectroscopy. Shaping of the pulses allows for adapting the electric field to a specific quantum system. The optimal pulse shape is often found in closed feedback loop optimizations. ${ }^{4}$ This technique of coherent control has been successfully applied in the

Institut für Experimentalphysik, Freie Universität Berlin, Arnimallee 14,D-14195 Berlin,Germany.E-mail:Weise.Fabian@gmail.com investigation and on the control of reaction mechanisms of various fields. ${ }^{5,6}$ Extending the modulation of the electric field by the polarization increased the controllability which was exemplified on different quantum mechanical systems. ${ }^{7-10}$

In this paper, we introduce the application of coherent control methods on pulses which have been transmitted through an optical fiber. We demonstrate a procedure to generate ultrashort pulses which are shaped in phase, amplitude, and polarization after propagation through a hollow core photonic crystal fiber. The pulses are controlled using a parametric encoding in which the individual sub-pulse parameters intensity, distance in time, phase, and chirps as well as the polarization including orientation, ellipticity and helicity are individually set. This parametric pulse shaping procedure in combination with a pre-compensation of the distortion introduced by the fiber is implemented in a closed feedback loop optimization of multi-photon ionization. The potassium dimer which has been thoroughly investigatedexperimentally and theoretically — serves as a model system. ${ }^{7,11,12}$ Furthermore, the shaped pulses are used in shaper-assisted pump-probe spectroscopy. The Fourier analysis of the resulting transients support the interpretation of the optimal pulse sequences which are obtained by the optimization including the role of the polarization.

\section{Experimental setup}

A schematic of the experimental setup is depicted in Fig. 1. The laser pulses of $2.5 \mu \mathrm{J}$ pulse energy are provided by a regenerative amplifier (RegA 9050, Coherent) with a repetition rate of $250 \mathrm{kHz}$. The spectral width amounts to $22 \mathrm{~nm}$ (FWHM) at a central wave length of $805 \mathrm{~nm}$. These pulses are modulated by a pulse shaper which is similar to the 


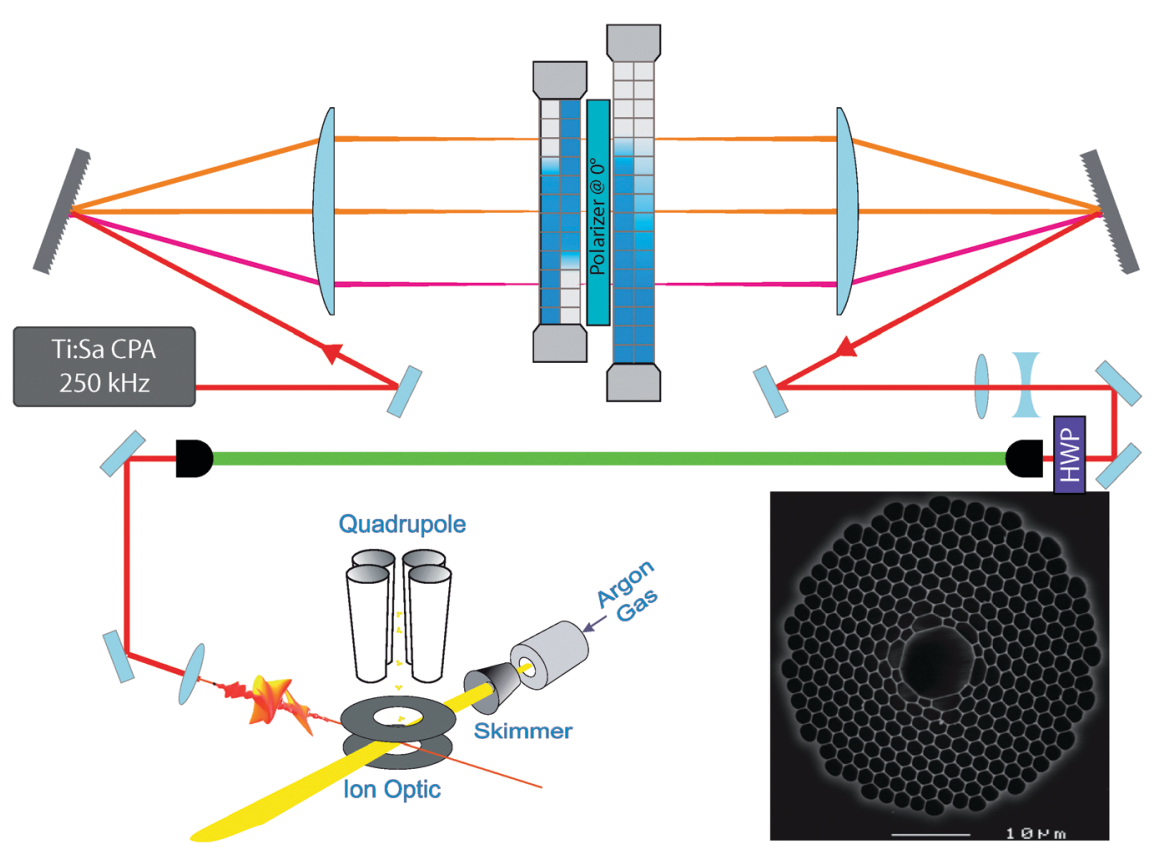

Fig. 1 Experimental setup: the ultrashort laser pulses are shaped in phase, amplitude, and polarization in the Fourier plane of a 4-f-line using two double liquid crystal spatial light modulators. They are coupled into a hollow core photonic crystal fiber with the help of a telescope, a half-wave plate, and a collimator. Then, the pulses are focused on a supersonic molecular beam. The created ions are mass selectively detected by a quadrupole followed by a secondary electron multiplier. The inset shows a micrograph of the used fiber. Taken from ref. 15 .

shaper setup presented in ref. 13. It consists of a 4-f-line with cylindrical lenses of $f=200 \mathrm{~mm}$ and gratings of 1200 grooves per millimeter. Two standard double liquid crystal modulators (SLM-256 and SLM-640, CRi) with the optical axis oriented at $\pm 45^{\circ}$ are placed in the Fourier plane. The two subsequent modulators are separated by a horizontally aligned polarizer. This setup is able to modulate the phase, amplitude, and polarization simultaneously and independently. In this setup, the polarization control is restricted to pulses with a fixed horizontal and vertical orientation of the principle axis of the electric field ellipse. This restriction is not obstructive since all sub-pulses that are used are linearly polarized, oriented in horizontal or vertical direction. The pulses are parametrically shaped using a sub-pulse encoding which is introduced in ref. 14. The intuitive sub-pulse parameters intensity, distance in time, ellipticity, phase, and chirps of each sub-pulse can be individually controlled.

The shaped pulse is coupled into a photonic crystal fiber using a telescope and a collimator. Employing these standard components we achieve a transmission efficiency of about $10 \%$. The fiber is a micro-structured single-mode hollow core fiber (HC-800-01, NKT Photonics) measuring $110 \mathrm{~cm}$ in length. More than $95 \%$ of the transmitted light is guided through the hollow core which is surrounded by a structure of 12 hollow hexagons of silica. This reduces non-linear effects and allows for transmitting ultrashort pulses of high energy. The cross sectional area of the core of this particular fiber measures $9.2 \mu \mathrm{m}$ for the short axis and $9.5 \mu \mathrm{m}$ for the long axis. The ends of the fiber are spliced onto thin glass windows of $100 \mu \mathrm{m}$ thickness and are connectorized by $\mathrm{PC} / \mathrm{FC}$ plugs. The fiber is transparent for a broad band of wavelengths $(>70 \mathrm{~nm}$ ) centered at $830 \mathrm{~nm}$. In this range the dispersion curve is nearly flat whereas the zero dispersion wavelength is situated at $805 \mathrm{~nm} .{ }^{15}$ For the pulse energies used in this work non-linear effects like self-phase modulation do not occur. The spectrum of the pulse is not distorted by the transmission through the fiber. These properties are in detailed investigated in ref. 16 and 17 .

After transmission through the fiber, the shaped pulses are focused on a molecular beam with a lens of $300 \mathrm{~mm}$ focal length. The supersonic beam is produced by co-expansion of potassium vapor at temperatures of approximately $800 \mathrm{~K}$ and argon with a pressure of 3.5 bar through a nozzle of $70 \mu \mathrm{m}$ into the vacuum. The beam mainly consists of potassium dimers which reside in their vibrational ground state. ${ }^{11}$ After interaction with the laser pulses the produced ions are mass-selected with a quadrupole filter and detected by a secondary electron multiplier. The ion signal of the ${ }^{39,39} \mathbf{K}_{2}$ isotope is investigated by pump-probe spectroscopy and serves as a fitness for an evolutionary algorithm in a closed feedback loop optimization.

\section{Parametric pulse generation}

The asymmetry of the core geometry as well as external and internal stress result in a birefringence of the fiber. Therefore, the fiber has two perpendicular axes with different dispersions. ${ }^{18}$ After propagation through the fiber this results in a temporal separation of the components. Further, each component is chirped differently. Pulses which enter the fiber linearly polarized in direction of either axis maintain their linear polarization. To generate pulses with a determined temporal shape and a defined state of polarization the axes of the fiber are aligned parallel to the principle axes of the electric field which are defined by the pulse shaper using a half-wave plate. For each axis a compensation for phase, time delay, and chirps 
is determined. Firstly, the chirp is compensated using quadratic, cubic, and 4th order phases in order to obtain a short pulse. Secondly, both pulses are shifted in time by a linear phase to achieve temporal overlap between them. Thirdly, the relative phase between the two pulses is adjusted to generate a single linearly polarized output pulse oriented at $45^{\circ}$. The Taylor terms amount $b_{0}=0, b_{1}=400 \mathrm{fs}, b_{2}=-7.00 \times 10^{3} \mathrm{fs}^{2}, b_{3}=$ $-1.46 \times 10^{6} \mathrm{fs}^{3}, b_{4}=-33.00 \times 10^{6} \mathrm{fs}^{4}$ for the $x$ component and $b_{0}=-0.25, b_{1}=-765.72 \mathrm{fs}, b_{2}=8.83 \times 10^{3} \mathrm{fs}^{2}, b_{3}=$ $-0.78 \times 10^{6} \mathrm{fs}^{3}, b_{4}=14.98 \times 10^{6} \mathrm{fs}^{4}$ for the $y$ component respectively. These parameters serve as an offset for the $x$ and $y$ pulse, respectively. Having this compensation determined, the pulse which is transmitted through the fiber can be arbitrarily controlled in phase, amplitude, and polarization. By changing the relative amplitudes of the $x$ and $y$ pulses the orientation of the pulse can be changed. The shift of the relative phase between the $x$ and $y$ components results in a change in ellipticity. Adding the desired chirp and delay on the compensation parameters controls the temporal shape and the position in time of the shaped pulse. This procedure can be extended to pulse sequences consisting of a variable number of sub-pulses. Any individual sub-pulse is generated by two pulses whose energy ratio, relative phase, and delay is set and the respective compensation is added. A detailed description can be found in ref. 16 .

\section{Feedback loop optimization}

In the feedback loop optimization the evolutionary algorithm controls the sub-pulse parameters of a triple pulse including the compensation parameters. This drastically reduces the search space and results in simpler solutions of the optimized pulse compared to a free optimization of the individual pixels. ${ }^{19}$ In one series, the three sub-pulses are all parallel oriented (denoted as XXX), in the other series the pulse in between was perpendicularly polarized respective to the outer ones (XYX).The pulses are all set to linear polarization with equally distributed energy. In optimizations of multi-photon processes a short pulse is often found to be the optimal solution. This solution is trivial and does not exhibit any dynamics of the system. In order to avoid an artifact originated intense short pulse as the final solution the algorithm was restricted in the range of distances and allowed chirps. The second pulse in the center of the pulse sequence was fixed at time zero. The first and the last pulse can be shifted in a time window of -1000 fs to $-200 \mathrm{fs}$ and +200 to $+1000 \mathrm{fs}$, respectively. The chirp of all three pulses was restricted in the limits $\pm 3 \times 10^{3} \mathrm{fs}^{2}$ for the linear chirp, $\pm 3 \times 10^{5} \mathrm{fs}^{3}$ for the quadratic chirp, and $\pm 3 \times 10^{6} \mathrm{fs}^{4}$ for the third order chirp. The phase of each sub-pulse was optimized in the whole range from $-\pi$ to $+\pi$. Due to the reduced search space in this parameterization the algorithm converges after about 30 generations. The pulse energy of the optimal pulse sequences in the interaction region is in the range of 25 to $50 \mathrm{~nJ}$. The temporal shape of the optimized pulses are detected in a time resolved cross correlation scheme. ${ }^{14}$ The optimizations revealed several solutions whereby some of them are very similar.

The intensity evolution of the pulse sequences which are determined by integrating the cross correlation signal over all

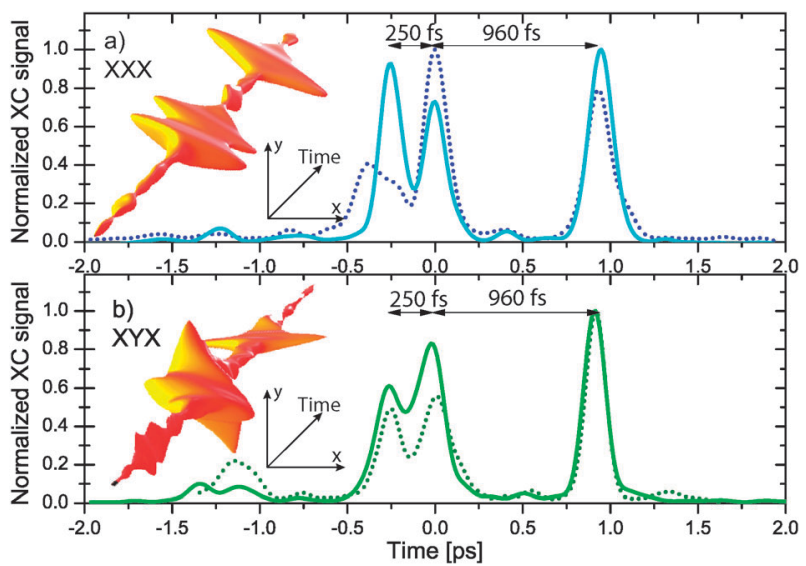

Fig. 2 Pulse sequence obtained by parametric feedback loop optimization. (a) depicts the sequence of three parallel polarized pulses. In (b) the polarization of the second pulse is perpendicular to the two outer ones. The inset shows a three dimensional representation of the pulses corresponding to the solid lines.

directions is depicted in Fig. 2 for both types of optimizations. To provide an intuitive picture of the optimally shaped pulse a three dimensional representation calculated from the measured data is presented. It shows clearly the different polarization obtained in each type of optimization. The similar and perseverative distances of the sub-pulses in the optimized pulse sequences are noticeable when comparing the time evolution of two different optimizations (solid and dotted traces). In both types of optimizations (XXX and XYX) the delay between the first and the second sub-pulse amounts approximately to $250 \mathrm{fs}$. The distance between the second and third pulses is optimized to about $960 \mathrm{fs}$. In spite of the similar cross correlation traces of the optimal pulses for the XXX and XYX optimizations the obtained fitness values differ.

The ion signal which is obtained by the optimal pulse is compared to the fitness of a short pulse of the same pulse energy. This linearly polarized pulse is about 62 fs long and likewise transmitted through the fiber before interacting with the molecular sample. In the case of the optimization of three parallel sub-pulses the recorded ion signal is $1.4 \pm 0.1$ times larger compared to the short pulse. For the pulse sequence in which the second pulse is perpendicularly oriented to the first and third pulses the factor amounts to $1.9 \pm 0.1$. The pulse which is additionally shaped in polarization is more efficient which can be understood by considering the orbital angular momentum of the participating electronic states of the dimer. In the excitation pathway, which is depicted in Fig. 4, $\Sigma$ and $\Pi$ states are involved. The transition dipole moment of a $\Sigma \leftarrow \Sigma$ transition is perpendicularly oriented to a $\Pi \leftarrow \Sigma$ transition. For an efficient excitation the first two subsequent excitation steps have to be perpendicularly polarized in an optimally shaped pulse. If the pulses are parallel polarized only the fraction of the transition dipole moment interacts which is projected onto the axis of the electric field oriented parallel to the preceding pulse. This leads to a reduced overall ionization efficiency. Another aspect is the simultaneous rotation of the molecule. ${ }^{20}$ When considering the ro-vibrational dynamics of 
the molecule the orientation of the dipole moment is turning to a distribution of different orientations with respect of the laser polarization during the propagation of the vibrational wave packet. This reduces the efficiency of the optimized shaped laser pulses. However, considering a rotational period of about 18 ps the dynamics is strongly governed by the faster vibrational dynamics. ${ }^{11}$

\section{Pump-probe spectroscopy}

Pump-probe spectra are measured to analyze the dynamics in the excited states. The pump-probe spectrum is recorded by pulses which have been propagated through the fiber. The pair of pump and probe pulses which are separated by the time delay $\Delta t$ is generated with the pulse shaper. Employing the described pulse shaper and the parameterization of a double pulse sequence make a mechanical delay stage obsolete. Moreover, no alignment is required to ensure a good spatial and temporal overlap of the pump and the probe pulse. Pump-probe spectra are recorded for two parallel pulses (XX) and for a perpendicular pair of pulses (XY). The time delay between the two pulses is scanned from $-2000 \mathrm{fs}$ to $+2000 \mathrm{fs}$ in steps of $8 \mathrm{fs}$. In order to remove the effect of any existent fluctuation of the molecular beam the average of several $\mathrm{XX}$ and $\mathrm{XY}$ types of pump-probe scans is taken. The transients show an oscillatory behavior for both parallel and perpendicular pairs of pump-probe pulses. The signal peaks for coincidence of the parallel pump and probe pulses. This is an artifact of the parameterization of the pulse sequence. When the two pulses are not delayed respective to each other the available pulse energy is nearly doubled. In order to determine the oscillation in the pump-probe scans a fast Fourier transformation is performed on the delay-dependent ion signal. To exclude the effects which occur at zero delay, the signal is separately processed for positive and negative delays. The Fourier spectrum for both types of pump-probe scans (XX and XY) is depicted in Fig. 3. The spectra of the XX and the $\mathrm{XY}$ pump-probe scans show frequency components
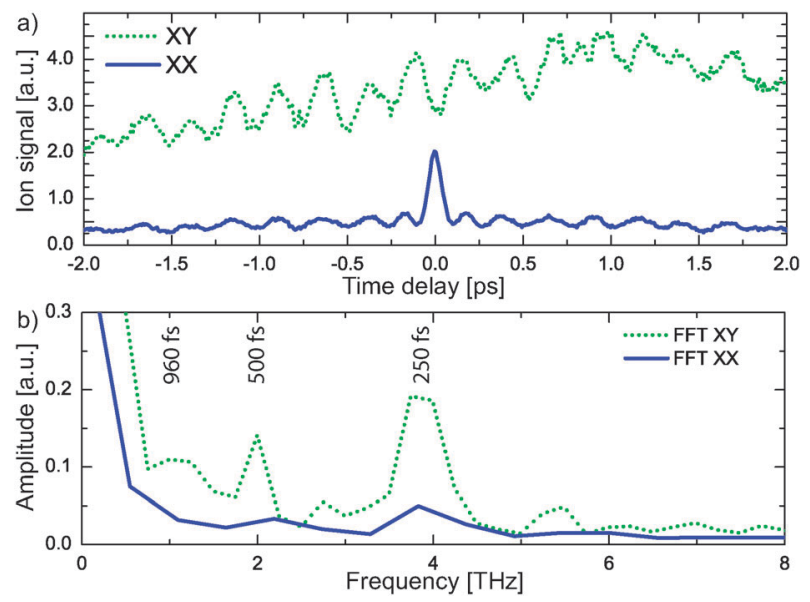

Fig. 3 (a) Transients obtained by pump-probe spectroscopy. The ion signal of parallel pump and probe pulses is compared to the case where pump and probe pulses are perpendicular. (b) Fourier transform of the transients.

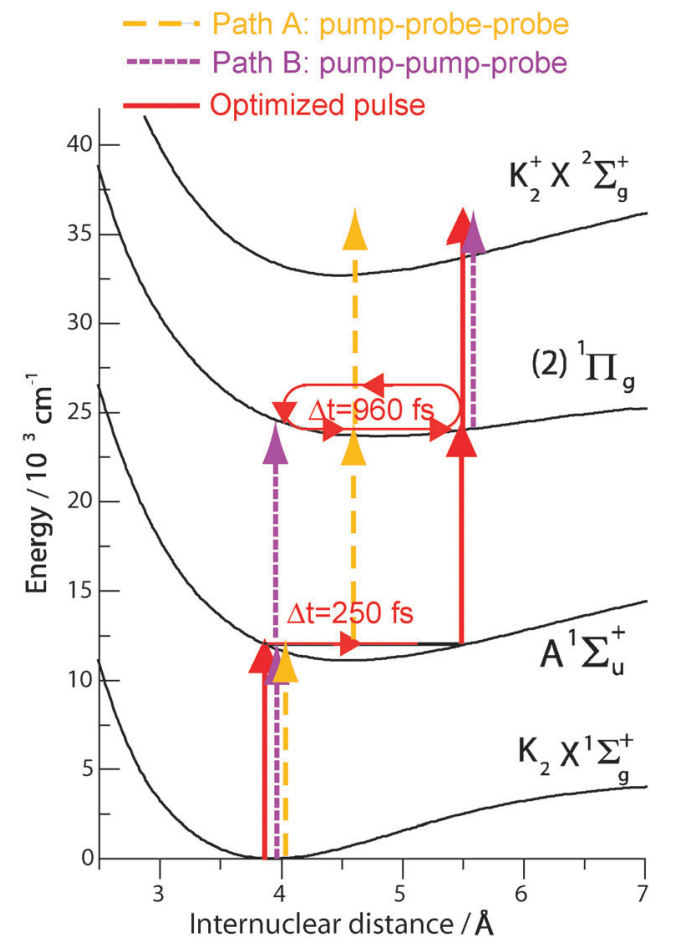

Fig. 4 Excitation scheme of the potassium dimer. The vertical red arrows indicate the ionization process induced by the pulses obtained by the feedback loop optimization. The broken lines indicate the excitation path in the pump-probe experiment. Path $\mathrm{A}$ is depicted by a long dashed yellow line and path B by a short dashed purple line. Potential energy curves are adapted from ref. 21 and 22.

which correspond to signal oscillation periods of $250 \mathrm{fs}$ and $500 \mathrm{fs}$. In the case of the pump-probe scan of two perpendicular pulses another frequency component which corresponds to an oscillation period of about 960 fs additionally contributes. The oscillation periods $250 \mathrm{fs}$ and $960 \mathrm{fs}$ are also found in the sub-pulse delays of the optimized pulses.

The proceeding excitation process is explained in the following. In the pump-probe scheme the ionization is achieved in two competing excitation pathways A and B which contribute to the ion signal ( $c f$. Fig. 4). Path A (pump, probe, probe): the first pulse creates a wavepacket in the $\mathrm{A}^{1} \Sigma_{\mathrm{u}}^{+}$state. In this first excited state, the wavepacket oscillates with a period of $500 \mathrm{fs}$ before it is transferred to the ionic state by a resonant two-photon transition. The transient Franck Condon point is strongly dependent on the wavelength of the exciting pulse. For the used wavelength the resonance condition yields a transition point which is situated before the classical turning point of the wavepacket. Therefore, the wavepacket crosses this transition point twice in one oscillation. The equidistant order of the modulation suggests a transition point which is situated temporally symmetric in between the classical turning points. This results in a doubled frequency in the modulation of the ion signal in the pump-probe trace. ${ }^{12}$ The distance of the first maxima to zero delay of the two pulses is with approximately 120 to 170 fs shorter than the oscillation period in the transient which supports the explanation with the central transition point. In the pathway B (pump, pump, 
probe) a wavepacket is created in the second excited state $2^{1} \Pi_{\mathrm{g}}$ by a two photon transition starting from the ground state. The population of higher lying vibrational states are favored by the broad band pulse due to larger Franck Condon factors resulting in a round trip time of $960 \mathrm{fs}$ for the wavepacket. This wavepacket is then transferred to the ionic state which is more likely at the outer turning point due to the R-depending dipole moment. ${ }^{12}$

\section{Excitation pathway}

With this information of the excited state dynamics the pathway of the optimized pulse can be revealed. It is marked by the red arrows in the excitation scheme which is depicted in Fig. 4. The solid vertical lines indicate the one-photon transitions. The first pulse creates a wavepacket in the $\mathrm{A}^{1} \Sigma_{\mathrm{u}}^{+}$ state. After 250 fs this population is transferred to the (2) ${ }^{1} \Pi_{\mathrm{g}}$ state by the second pulse. The transition at the center is not allowed due to the temporal restrictions in the parameterization. In this (2) ${ }^{1} \Pi_{\mathrm{g}}$ state, the wavepacket cycles one full period before it is transferred to the ionic state. This explains the delay of 960 fs between the second and the third sub-pulse. Additionally, two-photon transitions might occur. The fraction in the ion signal which is produced by the two photon transitions is not expected to contribute since the probability of these processes is much smaller.

\section{Conclusion and outlook}

In conclusion, we presented a new method to generate arbitrary polarization-shaped ultrashort pulses which are available after propagation through a fiber. These shaped pulses can be used for coherent control which is exemplified on the molecular test system $\mathrm{K}_{2}$. The pulses are parameterized in a sub-pulse encoding in which the intuitive physical parameters of each sub-pulse are controlled. This encoding was implemented in a closed feedback loop experiment which was applied on the multi-photon ionization of the potassium dimer. Further, this encoding enables us to perform out shaper-assisted phase stable pump-probe spectroscopy. Using these two methods the excitation pathway in the ionization process was revealed. In particular, the relevance of a polarization change within the optimized pulse shape is demonstrated.

The employment of photonic crystal fibers in combination with ultrafast pulse shaping techniques will be of practical use in different fields especially in biophysics and medicine. It allows us to separate the generation of pulses and the specific target or instrumentation and to transfer shaped pulses to any desired point even if it is difficult to access. This technique could be of beneficial use for in vivo analysis by multi-photon microscopy or photodynamic therapies. ${ }^{23-26}$ Further, the confined intense light field can be used in near-field microscopy providing femtosecond time resolution. ${ }^{27}$

\section{Acknowledgements}

We acknowledge Monika Pawłowska for discussion and Prof. Ludger Wöste for support. This work was financed by the Deutsche Forschungsgemeinschaft in the frame of the $\mathrm{Sfb} 450$.

\section{References}

1 S. H. Lee, A. L. Cavalieri, D. M. Fritz, M. Myaing and D. A. Reis, Adaptive dispersion compensation for remote fiber delivery of near-infrared femtosecond pulses, Opt. Lett., 2004, 29(22), 2602-2604.

2 P. Russell, Photonic Crystal Fibers, Science, 2003, 299(5605), 358-362.

3 A. A. Ishaaya, C. J. Hensley, B. Shim, S. Schrauth, K. W. Koch and A. L. Gaeta, Highly-efficient coupling of linearly- and radiallypolarized femtosecond pulses in hollow-core photonic band-gap fibers, Opt. Express, 2009, 17(21), 18630-18637.

4 R. S. Judson and H. Rabitz, Teaching lasers to control molecules, Phys. Rev. Lett., 1992, 68, 1500.

5 P. Nuernberger, G. Vogt, T. Brixner and G. Gerber, Femtosecond quantum control of molecular dynamics in the condensed phase, Phys. Chem. Chem. Phys., 2007, 9, 2470-2497.

6 C. Brif, R. Chakrabarti and H. Rabitz, Control of quantum phenomena: past, present and future, New J. Phys., 2010, 12(7), 075008.

7 T. Brixner, G. Krampert, T. Pfeifer, R. Selle, G. Gerber, M. Wollenhaupt, O. Graefe, C. Horn, D. Liese and T. Baumert, Quantum control by ultrafast polarization shaping, Phys. Rev. Lett., 2004, 92, 208301.

8 T. Suzuki, S. Minemoto, T. Kanai and H. Sakai, Optimal control of multiphoton ionization processes in aligned $\mathrm{I}_{2}$ molecules with time-dependent polarization pulses, Phys. Rev. Lett., 2004, 92, 133005.

9 F. Weise, S. M. Weber, M. Plewicki and A. Lindinger, Application of phase, amplitude and polarization shaped pulses for optimal control on molecules, Chem. Phys., 2007, 332, 313-317.

10 M. Aeschlimann, M. Bauer, D. Bayer, T. Brixner, F. J. G. de Abajo, W. Pfeiffer, M. Rohmer, C. Spindler and F. Steeb, Adaptive subwavelength control of nano-optical fields, Nature, 2007, 446(7133), 301-304.

11 S. Rutz, R. de Vivie-Riedle and E. Schreiber, Femtosecond wave-packet propagation in spin-orbit-coupled electronic states of ${ }^{39,39} \mathrm{~K}_{2}$ and ${ }^{39,41} \mathrm{~K}_{2}$, Phys. Rev. A: At., Mol., Opt. Phys., 1996, 54(1), 306-313.

12 C. Nicole, M. A. Bouchène, C. Meier, S. Magnier, E. Schreiber and B. Girard, Competition of different ionization pathways in $k_{2}$ studied by ultrafast pump-probe spectroscopy: A comparison between theory and experiment, J. Chem. Phys., 1999, 111(17), 7857-7864.

13 M. Plewicki, S. M. Weber, F. Weise and A. Lindinger, Independent control over the amplitude, phase and polarization of femtosecond pulses, Appl. Phys. B: Lasers Opt., 2007, 86, 259-263.

14 S. M. Weber, M. Plewicki, F. Weise and A. Lindinger, Parametric polarization pulse shaping demonstrated for optimal control of NaK, J. Chem. Phys., 2008, 128, 174306.

15 NKT Photonics. Data sheet: Hollow Core Photonic Bandgap Fiber HC-800-01.

16 F. Weise, G. Achazi and A. Lindinger, Parametrically polarizationshaped pulses via a hollow-core photonic crystal fiber, Phys. Rev. A: At., Mol., Opt. Phys., 2010, 82(5), 053827.

17 F. Weise, G. Achazi, M. Pawłowska and A. Lindinger, Systematic variation of parametrically shaped sub-pulse sequences after transmission through a photonic crystal fiber, Opt. Commun., 2011, in press, DOI : 10.1016/j.optcom.2011.02.026.

18 G. Bouwmans, F. Luan, J. Knight, P. S. J. Russell, L. Farr, B. Mangan and H. Sabert, Properties of a hollow-core photonic bandgap fiber at $850 \mathrm{~nm}$ wavelength, Opt. Express, 2003, 11(14), 1613-1620.

19 T. Hornung, R. Meier and M. Motzkus, Optimal control of molecular states in a learning loop with a parameterization in frequency and time domain, Chem. Phys. Lett., 2000, 326(5-6), 445-453.

20 T. Hornung and R. de Vivie-Riedle, Molecular population control including rotation, Europhys. Lett., 2003, 64(5), 703.

21 G. Jong, L. Li, T. J. Whang, W. C. Stwalley, J. A. Coxon, M. Li and A. M. Lyyra, $\mathrm{Cw}$ all-optical triple-resonance spectroscopy 
of $\mathrm{K}_{2}$ : Deperturbation analysis of the $\mathrm{a}^{1} \sigma_{\mathrm{u}}^{+}(v \leq 12)$ and $\mathrm{b}^{3} \pi_{\mathrm{u}}$ $(13 \leq v \leq 24)$ states, J. Mol. Spectrosc., 1992, 155(1), 115-135.

22 R. de Vivie-Riedle, B. Reischl, S. Rutz and E. Schreiber, Femtosecond study of multiphoton ionization processes in $\mathrm{k}_{2}$ at moderate laser intensities, J. Phys. Chem., 1995, 99, 16829-16834.

23 M. T. Myaing, D. J. MacDonald and X. Li, Fiber-optic scanning two-photon fluorescence endoscope, Opt. Lett., 2006, 31(8), 1076-1078.

24 K. König, Multiphoton microscopy in life sciences, J. Microsc., 2000, 200(2), 83-104.
25 K. T. Tsen, S.-W. D. Tsen, C.-L. Chang, C.-F. Hung, T.-C. Wu and J. G. Kiang, Inactivation of viruses with a very low power visible femtosecond laser, J. Phys.: Condens. Matter, 2007, 19(32), 322102.

26 S. B. Brown, E. A. Brown and I. Walker, The present and future role of photodynamic therapy in cancer treatment, Lancet Oncol., 2004, 5(8), 497-508.

27 B. A. Nechay, U. Siegner, M. Achermann, H. Bielefeldt and U. Keller, Femtosecond pump-probe near-field optical microscopy, Rev. Sci. Instrum., 1999, 70(6), 2758-2764. 